1998-03-01

\title{
Spatial resolution enhancement of SSM/I data
}

David G. Long

david_long@byu.edu

Douglas L. Daum

Follow this and additional works at: https://scholarsarchive.byu.edu/facpub

Part of the Electrical and Computer Engineering Commons

\section{Original Publication Citation}

Long, D. G., and D. L. Daum. "Spatial Resolution Enhancement of SSM/I Data." Geoscience and Remote Sensing, IEEE Transactions on 36.2 (1998): 47-17

\section{BYU ScholarsArchive Citation}

Long, David G. and Daum, Douglas L., "Spatial resolution enhancement of SSM/I data" (1998). Faculty Publications. 647.

https://scholarsarchive.byu.edu/facpub/647

This Peer-Reviewed Article is brought to you for free and open access by BYU ScholarsArchive. It has been accepted for inclusion in Faculty Publications by an authorized administrator of BYU ScholarsArchive. For more information, please contact ellen_amatangelo@byu.edu. 


\title{
Spatial Resolution Enhancement of SSM/I Data
}

\author{
David G. Long, Member, IEEE, and Douglas L. Daum
}

\begin{abstract}
One of the limitations in using Special Sensor Microwave/Imager (SSM/I) data for land and vegetation studies is the relatively low-spatial resolution. To ameliorate this limitation, resolution-enhancement algorithms can be applied to the data. In this paper, the Backus-Gilbert inversion (BGI) technique and the scatterometer image-reconstruction (SIR) algorithm are investigated as possible methods for creating enhanced resolution images from SSM/I data. The two algorithms are compared via both the simulation and the actual SSM/I data. The algorithms offer similar resolution enhancement, though SIR requires significantly less computation. Sample results over two land regions of South America are presented.
\end{abstract}

Index Terms-Deconvolution, resolution enhancement, SSM/I.

\section{INTRODUCTION}

$\mathbf{M}$ ICROWAVE radiometers, such as the Special Sensor Microwave/Imager (SSM/I) [6], [7], have wide application in atmospheric remote sensing over the ocean and provide essential inputs to numerical weather-prediction models. SSM/I data has also been used for land and ice studies, including snow-cover classification [5], measurements of soil and plant moisture content [9], [14], atmospheric moisture over land [11], land surface temperature [13], and mapping polar ice [19]. Unfortunately, the relatively lowresolution of the SSM/I is a limiting factor in its application for other land and ice studies. The intrinsic horizontal resolution of the SSM/I is determined by the antenna-beam pattern, and depending on the channel, its resolution varies from approximately 70 to $15 \mathrm{~km}$. To ameliorate this limitation, resolution-enhancement algorithms can be applied to the data [4], [16], [18]. Resolution enhancement is, in effect, equivalent to antenna-pattern deconvolution.

In this paper, we consider two resolution-enhancement algorithms developed for microwave data. The first, based on the Backus-Gilbert inversion (BGI) method, has been applied to $\mathrm{SSM} / \mathrm{I}$ data for both resolution enhancement and optimal interpolation [4], [15], [16], [18]. The second, known as the scatterometer image reconstruction (SIR) algorithm [12], was originally developed for SEASAT-A scatterometer data, but has been adapted for use with SSM/I data. In this paper, we compare the performance of these two algorithms for generating enhanced resolution brightness images from SSM/I data over land areas.

This paper is organized as follows: after a brief background discussion in Section II, Section III provides a detailed description of the BGI and SIR algorithms. The results of

Manuscript received October 26, 1995; revised August 20, 1996.

The authors are with Brigham Young University, Provo, UT 84602 USA (e-mail: long@ee.byu.edu).

Publisher Item Identifier S 0196-2892(98)00550-6.
TABLE I

Sizes of the 3-dB AnTENNA FootPrints of the SSM/I Channels. The Approximate Spacing of the Measurements in the Cross-Track and Along-Track Directions (i.e., the Spatial Sampling Density) Is Also Shown

\begin{tabular}{c|c|c|c|c}
\hline \multicolumn{2}{c|}{ SSM/I Channel } & \multicolumn{2}{c|}{$3 \mathrm{~dB}$ Footprint (km) } & $\begin{array}{c}\text { Approximate } \\
\text { Frequency (GHz) }\end{array}$ \\
\hline 19.35 & Pol. & Along-track & Cross-track & Spacing (km) \\
\hline 19.35 & $\mathrm{H}$ & 69 & 43 & 25 \\
\hline 22.235 & $\mathrm{~V}$ & 50 & 43 & 25 \\
\hline 37.0 & $\mathrm{~V}$ & 37 & 40 & 25 \\
\hline 37.0 & $\mathrm{H}$ & 37 & 28 & 25 \\
\hline 85.5 & $\mathrm{~V}$ & 15 & 29 & 25 \\
\hline 85.5 & $\mathrm{H}$ & 15 & 13 & 12.5 \\
\hline
\end{tabular}

simulations used to compare the performance of the algorithms are described in Section IV. Comparison of the algorithms using actual SSM/I data is given in Section V. Finally, conclusions are made in Section VI.

\section{BACKGROUND}

The SSM/I is a total-power, seven-channel, four-frequency radiometer [6]. The channels are vertical and horizontal polarizations at $19.35,37.0$, and $85.5 \mathrm{GHz}$ and vertical polarization at $22.235 \mathrm{GHz}$ (see Table I). Radiometric brightnesstemperature measurements are made with an integrate-anddump filter as the antenna scans the ground track [8]. The $3-\mathrm{dB}$ antenna footprints range from about $15-70 \mathrm{~km}$ in the cross-scan direction and $13-43 \mathrm{~km}$ in the along-scan direction (see Table I). The 3-dB antenna footprints, which are different for each frequency, generally have an elliptical shape on the surface of the Earth, due to the elevation angle of the radiometer beam [7].

The brightness temperatures observed by the SSM/I are a function of the effective brightness temperature of the Earth's surface and the emission, scattering, and attenuation of the atmosphere. Because of the spatial and temporal variability of the surface brightness, which is a function of the properties of the soil and overlaying vegetation and their physical temperatures, it is difficult to decompose the observed brightness into its individual components. The most crucial factors affecting a radiometric measurement, however, are the surface emissivity and temperature, the vegetation canopy, the viewing angle, and the atmospheric conditions [20].

For this research, we are primarily interested in the surface brightness temperature. Ignoring, for the moment, the effects of the atmosphere, an SSM/I measurement can be modeled as a product of the surface brightness and the antenna pattern. The $i$ th measurement $T_{a}(i)$ (in $K$ ) is obtained by integrating the product of the surface brightness response $T_{b}(x, y)$ (in $K$ ) 


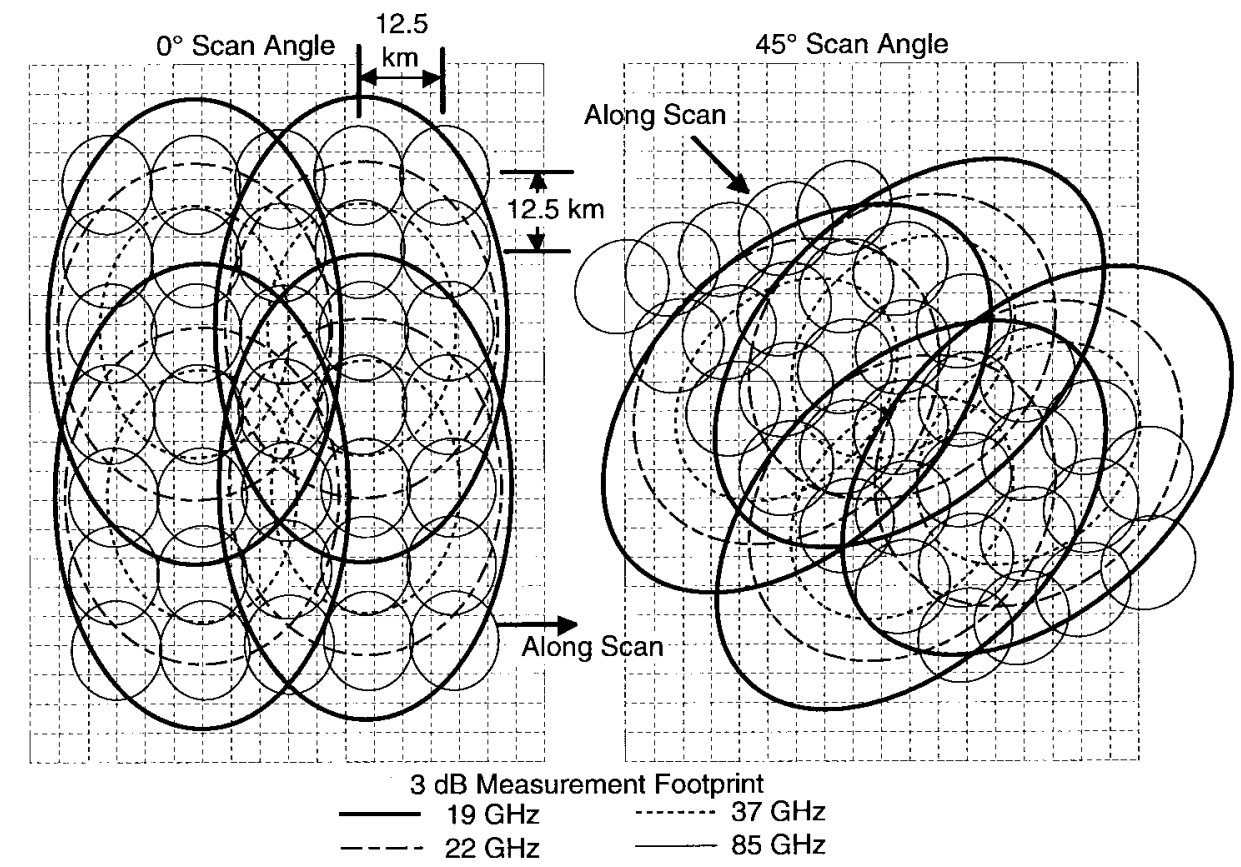

Fig. 1. Geometry of the resolution-enhancement algorithm. Enhanced resolution images are produced on the underlying rectilinear grid. The locations of the measurement footprints for several along-track measurements for two scans are illustrated for two different scan locations.

and the antenna at the surface $G_{i}(x, y)$

$$
T_{a}(i)=\bar{G}_{i}^{-1} \iint G_{i}(x, y) T_{b}(x, y) d x d y
$$

where

$$
\bar{G}_{i}=\iint G_{i}(x, y) d x d y
$$

where integrals over the surface area are corresponding to the nonnegligible gain of the antenna. The dependence of $G$ on $i$ arises from the boresight pointing of the antenna, which changes as the antenna scans the surface. Note that the antenna pattern acts as a low-pass filter of the surface brightness, limiting the effective resolution of the measurement.

\section{SSM/I RESOLUTION-ENHANCEMENT ALGORITHMS}

When using SSM/I data for land and vegetation studies, several limiting factors become apparent. These include the differing spatial resolutions of the channels, their lack of spatial coregistration, and the overall low-spatial resolution. To ameliorate some of these difficulties, a spatial resolutionenhancement algorithm can be applied to the data [4], [16], [18]. To this end, we consider two different resolutionenhancement algorithms in this paper: one based on the BGI and a new algorithm based on the SIR algorithm. These are briefly described below. Their performance for land imaging are compared using both the simulated and the actual data in later sections.

Note that both the BGI and SIR resolution-enhancement algorithms generate images from the radiometer measurements. While they effectively deconvolve the antenna pattern, the algorithms do not "enhance" previously-produced images, as done by image-processing algorithms; rather, they are "reconstruction" algorithms, which attempt to reconstruct the underlying brightness distribution $\left(T_{b}\right)$ on a high-resolution grid (see Fig. 1) from the lower-resolution measurements $\left(T_{a}\right)$ [12].

While the grid can be made arbitrarily fine, the resolutionenhancement capabilities of both the BGI and SIR algorithms are dependent on the sampling pattern and the overlap in the response functions of the measurements. In general, the higher the sampling density (leading to more overlap in the measurements), the better the resolution enhancement. Note that an overly fine grid increases the computational requirements without improving the effective resolution of the output image. Like all resolution-enhancement techniques, both algorithms provide improved resolution at the expense of an increased noise level in the images.

\section{A. The BGI Method}

The BGI algorithm is an inversion method for solving integral equations [1]-[3]. The algorithm is used to determine surface brightness from integrated, overlapping antenna patterns. Several authors, including Farrar and Smith [4], Poe [15], Robinson et al. [16], and Stogryn [18], have used BGI to successfully enhance the spatial resolution and/or to perform optimal interpolation of SSM/I data to either raise or lower resolution for use in multichannel studies. They address the utility of resolution enhancement for SSM/I measurements.

When employed for spatial resolution enhancement, the BGI algorithm produces a weighted least-squares estimate of the surface brightness on a rectilinear surface grid finer than the intrinsic resolution of the sensor (see Fig. 1). Given a set of antenna temperature measurements $\left\{T_{a}(i)\right\}$ with associated antenna gain patterns $G_{i}(x, y)$, the algorithm estimates the brightness temperatures $T_{b}\left(x_{j}, y_{j}\right)$ for each pixel $\left(x_{j}, y_{j}\right)$ of the enhanced resolution image. 
To estimate $T_{b}$ for a given pixel, the BGI method uses a linear combination of $N$ "nearby" measurements, i.e.,

$$
T_{b}\left(x_{j}, y_{j}\right)=\sum_{i=1}^{N} a_{i j} T_{a}(i)
$$

where the coefficients $a_{i j}$ are determined from the measurement geometry and the noise-correlation matrix [4], [15]. Note that these coefficients are different for every pixel, due to the varying antenna geometry over the swath.

There is no unique solution for the coefficients $a_{i j}$; however, regularization permits a subjective tradeoff between the noise level in the image and in the resolution. Regularization and selection of the tuning parameters are described in [3] and [18], but we provide some comments here. There are two tuning parameters, the dimensional parameter $\omega$ and the noisetuning parameter $\gamma$. The dimensional parameter $\omega$ is arbitrary, but does affect the optimum value of $\gamma$. Following Robinson et al. [16], the dimensional-tuning parameter $\omega$ is set to 0.001 . The noise-tuning parameter $\gamma$, which can vary from 0.0 to $\pi / 2$, controls the tradeoff between the resolution and the noise parameter. The value of $\gamma$ can be subjectively selected to optimize the resulting image. The value of the optimum $\gamma$ is dependent on the value of $\Delta T$, used for the noise level as well as the penalty and the reference functions. We have used the constant penalty function of Farrar and Smith [4]. To maximize the resolution enhancement, the reference function is defined to be unity over the pixel of interest and zero elsewhere.

In general, $\gamma$ is different for each SSM/I channel. Farrar and Smith [4] developed an objective technique for selecting $\gamma$ for the 19-, 22-, and 37-GHz SSM/I channels, based on maximizing the correlation between the $85-\mathrm{GHz}$ channels and the particular channel of interest. However, for a single-channel instrument or for the $85-\mathrm{GHz}$ channels, $\gamma$ must be subjectively chosen. A detailed description of the BGI algorithm is given by Poe [15], Robinson et al. [16], and Stogryn [18].

The BGI-produced image is affected by the definition of "nearby" $(N)$ and the relative locations and gain patterns of the measurements included in the sum in (3). Restricting the size of the local region defining "nearby" measurements reduces the computational load at the expense of accuracy. Increasing the size of the local area (and $N$ ) to include additional measurements in (3) can improve the accuracy of the resolution enhancement, but may significantly increase the computational load. Previous investigators have used a fixed range of angles around the antenna-pointing direction to define "nearby" [18]. This approach can lead to numerical problems in the matrix-inversion step of the algorithm if the angle range is large enough to include directions with very small gain. To avoid this problem, a somewhat different approach is used in this paper. We define "nearby" measurements as those measurements that have nonnegligible gain at the pixel of interest. A threshold, denoted by $g_{c}$, is used to determine if the gain is nonnegligible: the measurement is used in (3) only if the relative antenna gain at the pixel of interest is greater than the threshold. A $g_{c}$ of $11 \mathrm{~dB}$ below the peak gain has been used in this paper [18]. Setting a lower gain threshold results in more measurements being used in (3), but increases the noise level of the images. The exact number of "nearby" measurements used at a given pixel is a function of the gain pattern and sampling geometry and varies across the swath and for each channel.

\section{B. The SIR Algorithm}

The SIR algorithm, originally designed to produce multivariate scatterometer images [12], has been adapted for radiometer measurements. It produces radiometric images by using an iterative procedure from an initial-brightness estimate. The procedure is nonlinear and depends on the antennapattern dimension, shape, and measurement overlap to obtain resolution enhancement.

The SIR algorithm is a variation of the multiplicative algebraic-reconstruction technique (MART), a maximumentropy reconstruction method [10]. The detailed derivation for scatterometer data is found in Long et al. [12]. Since the radiometer version of the algorithm has not been previously published, we provide it in its entirety below.

SIR provides a maximum-entropy estimate of the brightness temperature for each element of a rectilinear grid of pixels. Assuming that the brightness temperature is constant within each pixel, (1) can be written as

$$
T_{a}(i)=\frac{1}{\bar{G}_{i}} \sum_{x=L_{i}}^{R_{i}} \sum_{y=B_{i}}^{T_{i}} T_{b}(x, y) G_{i}(x, y)
$$

where

$$
\bar{G}_{i}=\sum_{x=L_{i}}^{R_{i}} \sum_{y=B_{i}}^{T_{i}} G_{i}(x, y)
$$

and $L_{i}, R_{i}, B_{i}$, and $T_{i}$ define a bounding rectangle for the $i$ th measurement.

In implementing the SIR algorithm, an initial guess for the $T_{b}$ image is first made: typically, the average expected brightness temperature. A predicted value (forward projection) of each measurement is calculated from a current estimate of $T_{b}$ and is compared to the measurement. A scale factor is then computed as the ratio of the measurement to the forward projection. An update term is computed for each pixel in the measurement cell by multiplying by the scale factor. Between iterations, each pixel in the image is updated by averaging the update terms for the pixel. As the process iterates, the scale factors approach unity and all of the forward projections match the measurements. When noise is present in the measurements, however, the scale factors may not always converge to unity. In this case, the algorithm attempts to balance the various scale factors for each measurement and pixel-using maximum entropy. In SIR, the scale factors calculated for each forward projection are damped by taking the square root. In addition, the update terms are computed in a manner that limits the amount of change for a single update [see (8) below]. These steps tend to reduce the sensitivity of the update terms to noise.

Let $T_{b}(x, y)$ be denoted by $p_{j}$ and $G_{i}(x, y)$ by $h_{i j}$, where $j$ is the row-scanned pixel number and $i$ is the measurement number. Then for the $k$ th iteration and for the $i$ th measurement 
in the data set $z_{i}$ and its corresponding weighting function $h_{i j}$, the forward projection $f_{i}^{k}$ is calculated as

$$
\begin{aligned}
f_{i}^{k} & =\frac{1}{q_{i}} \sum_{n=1}^{M} h_{i n} p_{n}^{k} \\
q_{i} & =\sum_{n=1}^{M} h_{i n}
\end{aligned}
$$

where $M$ is the number of pixels in the image. The scale factor $d_{i}^{k}$ is then computed as

$$
d_{i}^{k}=\left[\frac{T_{a}(i)}{f_{i}^{k}}\right]^{1 / 2}
$$

The nonlinear update term $u_{i j}^{k}$ is then computed, according to

$$
u_{i j}^{k}= \begin{cases}{\left[\frac{1}{2 f_{i}^{k}}\left(1-\frac{1}{d_{i}^{k}}\right)+\frac{1}{p_{j}^{k} d_{i}^{k}}\right]^{-1},} & d_{i}^{k} \geq 1 \\ {\left[\frac{1}{2} f_{i}^{k}\left(1-d_{i}^{k}\right)+p_{j}^{k} d_{i}^{k}\right],} & d_{i}^{k}<1 .\end{cases}
$$

After the entire data set has been processed, each pixel estimate $p_{j}^{k}$ is updated by computing a weighted average of the update terms, i.e. ( $N_{m}$ is the number of measurements),

$$
\begin{aligned}
p_{j}^{k+1} & =\frac{1}{g_{j}} \sum_{i=1}^{N_{m}} h_{i j} u_{i j}^{k} \\
g_{j} & =\sum_{i=1}^{N_{m}} h_{i j} .
\end{aligned}
$$

This set of equations is iterated over $k$ for $N$ iterations until the scale factors approach unity. Because of the damping employed in the update terms, the algorithm always converges, and the iteration may be continued even for very large $k$ values. As shown in the next section, however, the accuracy of the algorithm at first increases with the iteration number, but then may decrease as the iteration continues. This is a common problem with algebraic reconstruction algorithms and is the result of excessive noise amplification. Fortunately, the algorithm's performance is not particularly sensitive to the exact number of iterations used, and a range of values will produce good results. This range depends on the antenna-pattern size and sampling density. In the next section, simulation is used to determine the "optimum" number of iterations $N$ to use for each channel.

\section{Simulation Comparison Between THE BGI AND SIR ALGORITHMS}

While BGI, in various forms, has previously been applied to SSM/I data [4], [15], [16], [18], SIR has not been previously applied to radiometer data. To compare the two algorithms and understand their weaknesses and strengths, we first utilize simulated measurements of known surface brightness distributions. For this, we have generated a synthetic image containing test patterns and characteristics similar to those observed over the Amazon Basin. We provide sample images for two channels: 19- and 37-GHz V-pol.

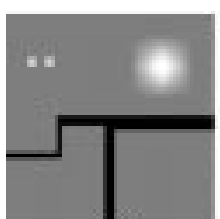

(a)

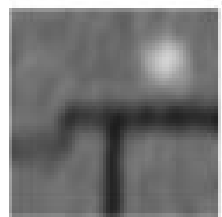

(e)

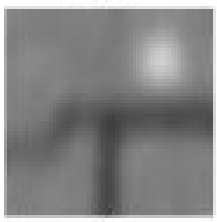

(i)

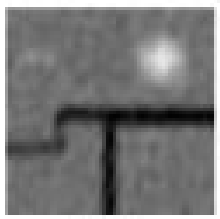

(m)

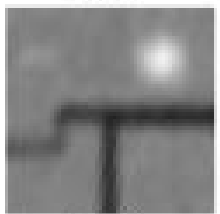

(q)

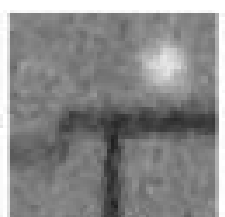

(b)

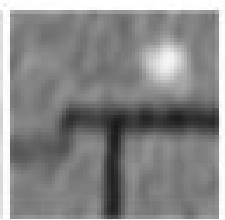

(f)

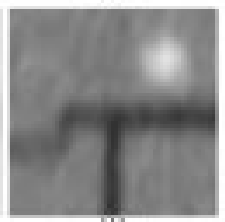

(j)

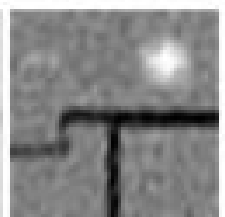

(n)

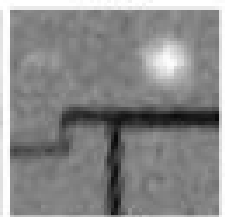

(r)

270

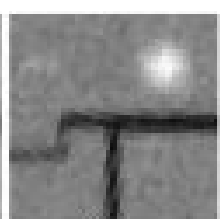

(c)

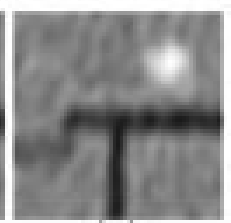

(g)

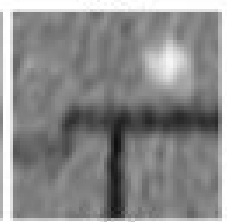

(k)

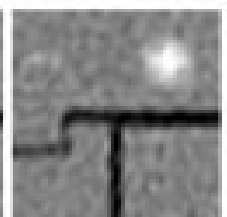

(o)

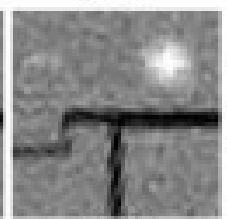

(s)

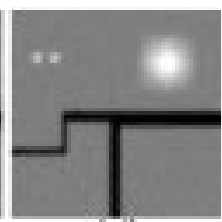

(d)

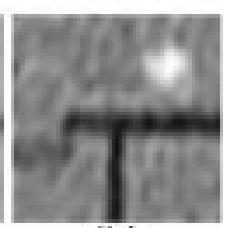

(h)

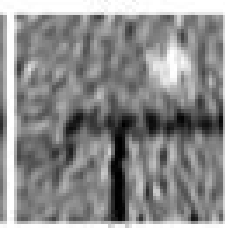

(1)

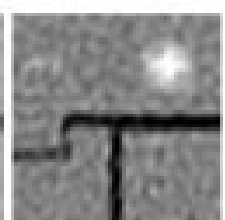

(p)

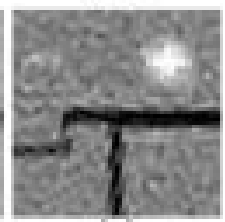

(t)
Fig. 2. Simulation images. Images cover an area $6^{\circ} \times 6^{\circ}$ in longitude and latitude at $32 \mathrm{pixels} / \mathrm{deg}$. (a) Synthetic test image, (b) nonenhanced $19 \mathrm{~V}$, (c) nonenhanced $37 \mathrm{~V}$, (d) nonenhanced $85 \mathrm{~V}$, (e) SIR $19 \mathrm{~V}, k=10$, (f) SIR $19 \mathrm{~V}, k=20$, (g) SIR $19 \mathrm{~V}, k=25$, (h) SIR $19 \mathrm{~V}, k=40$, (i) BGI $19 \mathrm{~V}$, $\gamma=\pi / 2$, (j) BGI $19 \mathrm{~V}, \gamma=7 / 5$, (k) BGI $19 \mathrm{~V}, \gamma=1$, (1) BGI $19 \mathrm{~V}, \gamma=$ 0 , (m) $\operatorname{SIR} 37 \mathrm{~V}, k=10$, (n) SIR $37 \mathrm{~V}, k=20$, (o) SIR $37 \mathrm{~V}, k=25$, (p) SIR $37 \mathrm{~V}, k=40$, (q) BGI $37 \mathrm{~V}, \gamma=\pi / 2$, (r) BGI $37 \mathrm{~V}, \gamma=7 / 5$, (s) BGI $37 \mathrm{~V}, \gamma=1$, and (t) BGI $37 \mathrm{~V}, \gamma=0$.

The synthetic test image [shown in Fig. 2(a)] is $6^{\circ}$ longitude $\times 6^{\circ}$ latitude in size and includes a number of features similar to those seen in radiometric data over the Amazon. The features include a $270-\mathrm{K}$ "river," $295-\mathrm{K}$ "spots," and a pyramid-like feature. The background temperature is $285 \mathrm{~K}$, similar to the average temperature from SSM/I data over the Amazon. The pixel resolution was arbitrarily chosen to be 32 pixels/deg (to be several times finer than the resolution of the measurements). To make the simulation as realistic as possible, simulated brightness measurements are generated with (1) using the SSM/I antenna pattern and sampling geometry from midswath for a single-ascending pass. Monte Carlo random noise is added to the simulated measurements.

Noise, in the sense used here, refers to the random variability in the observed brightness temperatures over a region. This variability arises from two sources: 1) radiometric measurement error $(\Delta T)$ and 2) subscale spatial variability in 
TABLE II

F10 SSM/I DATA Summary From $-55^{\circ}$ E To-52 $2^{\circ}$, $0^{\circ} \mathrm{N}$ To $3^{\circ} \mathrm{N}$, Descending Passes, SePtember 1992

\begin{tabular}{c|c|c|c|c}
\hline Channel & Ave $T_{b}(\mathrm{~K})$ & $\sigma(\mathrm{K})$ & Max $(\mathrm{K})$ & $\operatorname{Min}(\mathrm{K})$ \\
\hline $19.35 \mathrm{GHz} \mathrm{V}$ & 288.3 & 1.06 & 291.5 & 285.6 \\
\hline $19.35 \mathrm{GHz} \mathrm{H}$ & 287.6 & 1.05 & 290.5 & 284.3 \\
\hline $22.23 \mathrm{GHz} \mathrm{V}$ & 287.0 & 0.53 & 290.3 & 284.0 \\
\hline $37.00 \mathrm{GHz} \mathrm{V}$ & 284.1 & 0.76 & 287.0 & 281.4 \\
\hline $37.00 \mathrm{GHz} \mathrm{H}$ & 283.7 & 0.91 & 286.4 & 280.7 \\
\hline $85.50 \mathrm{GHz} \mathrm{V}$ & 286.2 & 0.33 & 289.4 & 282.5 \\
\hline $85.50 \mathrm{GHz} \mathrm{H}$ & 286.0 & 0.94 & 289.4 & 282.0 \\
\hline
\end{tabular}

the surface brightness temperature. To make the simulation noise as realistic as possible, the variability of the SSM/I measurements over the Amazon were examined. A large $\left(5^{\circ}\right.$ longitude $\times 3^{\mathrm{C}}$ latitude) spatially homogeneous region of the Amazon Basin was selected, and the variance of the brightness measurements determined. The results are summarized in Table II. The average brightness is in the mid $280-\mathrm{K}$ range, while the standard deviation is generally less than $1 \mathrm{~K}$.

For the noisy simulations, Gaussian noise, with a standard deviation given by the value of $\sigma$ in Table II, is added to the simulated measurements. In both the simulations and the actual data results shown below, this same $\sigma$ value is used for the standard deviation of the noise in the BGI algorithm, rather than just $\Delta T$.

\section{A. Simulation Results}

The simulation results for the 19- and 37-GHz V-pol channels are illustrated in Fig. 2. For comparison, nonenhanced 19-, 37-, and 85-GHz V-pol images are given in Fig. 2(b)-(d). These images were generated by assigning each pixel the measurement value corresponding to the measurement with the largest antenna gain.

The simulation results for different iterations $(k=10$, 20, 25, and 40) of SIR are illustrated in Fig. 2(e)-(h) (19$\mathrm{V}$ channel) and (m)-(p) (37-V channel). Simulation results for BGI for various values of the BGI-tuning parameter $\gamma$ are shown in Fig. 2(i)-(l) (19-V channel) and (q)-(t) (37$\mathrm{V}$ channel). Examining these images, we see that as SIR is iterated, the resolution improves, though at the expense of additional noise. Similarly, as $\gamma$ is varied from $\pi / 2$ (no resolution enhancement, maximum noise filtering) to $\gamma=0$ (maximum enhancement, no noise suppression), the resolution improves at the expense of additional noise.

To objectively compare the algorithms, we use the rms error and the correlation coefficient between pixel values of the reconstructed images and the truth image. Plots of the rms error and the correlation coefficient for all the channels versus the iteration number for SIR and $\gamma$ for BGI are contained in Fig. 3. We note that as the number of iterations for SIR is increased, the correlation first increases and then, for most channels, decreases somewhat as the iterations continue. Similarly, the rms error first decreases, then generally increases somewhat as the number of iterations continues as a result of noise amplification. The "optimum" number of iterations $N$ to use for SIR is selected as the $k$ value, which maximizes the correlation. For BGI, we choose the $\gamma$ that produces the highest correlation [4]. The "optimum" $N$ and $\gamma$ values for each
TABLE III

RMS ERror and Correlation Coefficient Between Simulated Images and the True Synthetic Image for Each Channel. The $N$ and $\gamma$ Values Resulting In the MaXimum Correlation Are Indicated. No Ocean or River Pixels Are Included in the Statistics Calculation

\begin{tabular}{c|c|c|c|c}
\hline Channel & Algorithm & $N$ or $\gamma$ & RMSE $(\mathrm{K})$ & Correlation Coef. \\
\hline $19 \mathrm{H}$ & non & - & 3.002 & 0.760 \\
& SIR & 25 & 2.701 & 0.810 \\
& BGI & 1 & 2.790 & 0.796 \\
\hline $19 \mathrm{~V}$ & non & - & 3.006 & 0.759 \\
& SIR & 25 & 2.703 & 0.809 \\
& BGI & 1 & 2.793 & 0.795 \\
\hline $22 \mathrm{~V}$ & non & - & 2.734 & 0.812 \\
& SIR & 40 & 2.362 & 0.860 \\
& BGI & $7 / 5$ & 2.499 & 0.843 \\
\hline $37 \mathrm{H}$ & non & - & 2.488 & 0.842 \\
& SIR & 15 & 2.250 & 0.879 \\
& BGI & $7 / 5$ & 2.332 & 0.866 \\
\hline $37 \mathrm{~V}$ & non & - & 2.431 & 0.851 \\
& SIR & 20 & 2.116 & 0.888 \\
& BGI & $7 / 5$ & 2.262 & 0.874 \\
\hline $85 \mathrm{H}$ & non & - & 1.807 & 0.920 \\
& SIR & 10 & 2.633 & 0.940 \\
& BGI & $\pi / 2$ & 1.868 & 0.914 \\
\hline $85 \mathrm{~V}$ & non & - & 1.569 & 0.941 \\
& SIR & 25 & 1.268 & 0.961 \\
& BGI & $3 / 2$ & 1.695 & 0.930 \\
\hline
\end{tabular}

channel are given in Table III, along with the corresponding correlation coefficients and the rms error. These values of $N$ and $\gamma$ are used in the remainder of this paper. The corresponding "optimum" images for SIR are Fig. 2(g) (19 V) and (n) (37 V). For BGI, the "optimum" images are Fig. 2(k) $(19 \mathrm{~V})$ and $(\mathrm{r})(37 \mathrm{~V})$.

Comparing the tabulated rms error and the correlation between the enhanced images and the truth image given in Table III, we conclude that the performance of SIR and BGI are broadly similar, though SIR has somewhat lower rms error and higher correlation. With the exception of $85 \mathrm{~V}$, the table also shows that both BGI and SIR produce improvement in the correlation coefficient over the nonenhanced image.

The "optimum" SIR and BGI images for each channel [Fig. 2(g) compared with (k) and (n) compared with (r)] are visually very similar. Both algorithms provide better definition of the river and other features than the nonenhanced image. The SIR image has fewer artifacts along the river and the edge of the pyramid than the BGI image. This is most evident at $37 \mathrm{GHz}$. Both algorithms are unable to accurately reconstruct the proper width of the river along its full course. The spots are not clearly discernible in any of the images, though there are hints of them in the images. The 37-GHz images have sharper edges for the river and for the pyramid, but have more "noise" in the smooth forest regions. Subjectively, the enhanced images provide better definition than the nonenhanced image. This, coupled with the improved correlation of the nonenhanced images, demonstrates that the resolution enhancement is effective for SSM/I.

One of the difficulties with using the actual data is that the true brightness distribution is not known. This makes quantitative evaluation of the resolution enhancement impossible. As a result, we must resort to comparing the enhanced resolution 


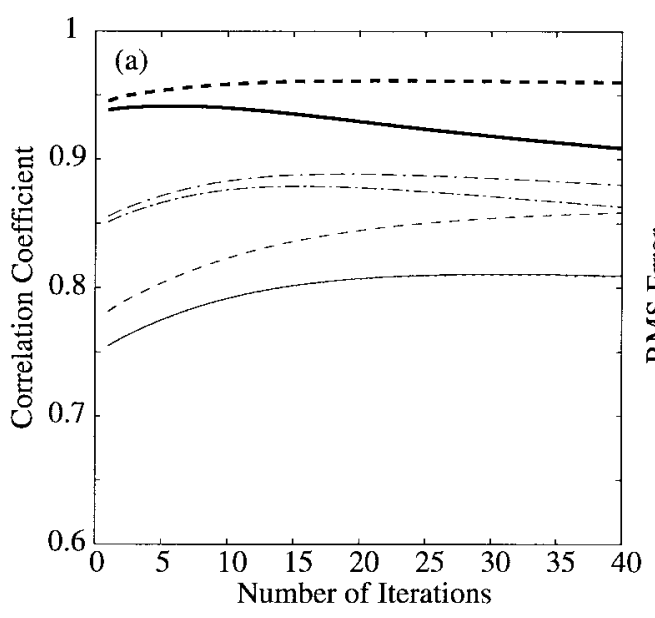

(a)

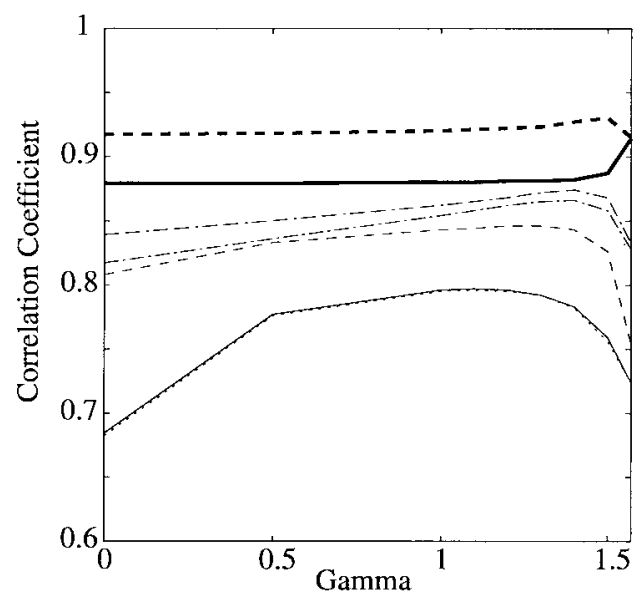

(c)

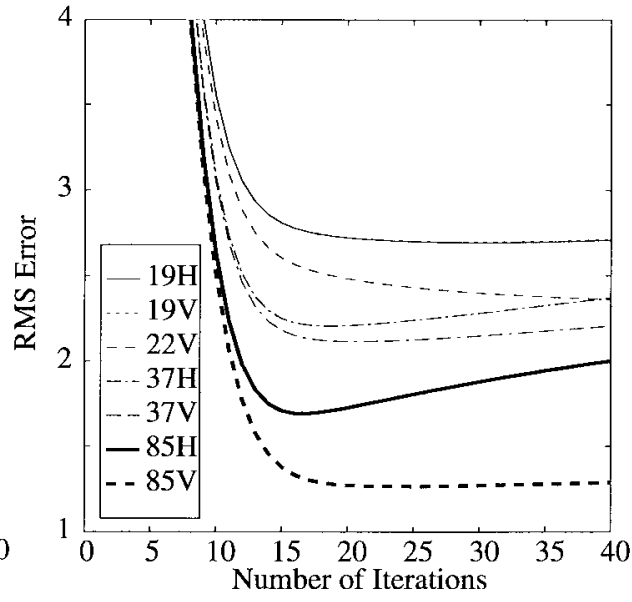

(b)

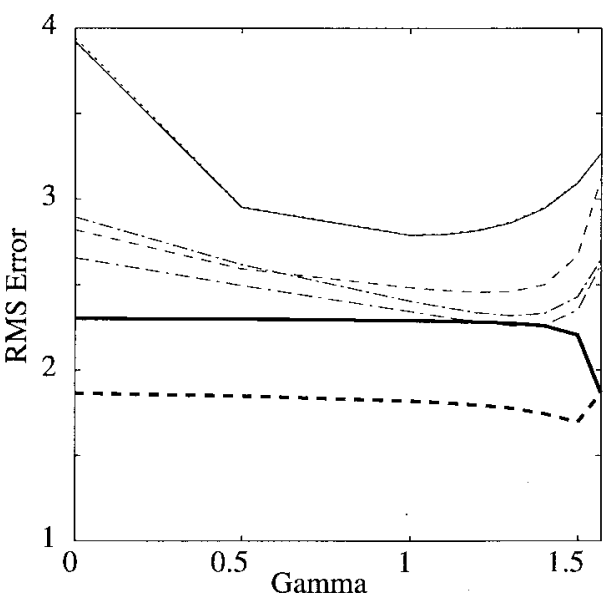

(d)

Fig. 3. Correlation coefficient and rms error between simulation results and true simulation images. (a) SIR 19-V correlation versus SIR iteration number $k$, (b) SIR 19-V rms error versus SIR iteration number $k$, (c) BGI 37-V correlation versus BGI $\gamma$ coefficient, and (d) BGI 37-V rms error versus BGI $\gamma$ coefficient. The $19-\mathrm{H}$ and $19-\mathrm{V}$ lines overlay each other.

images to the data from a higher frequency channel, which has higher intrinsic resolution. This comparison is complicated by the fact that since the two channels operate at different frequencies, the surface brightness-response characteristics may be different. Nevertheless, with this limitation in mind, the correlation between the two channels can provide a measure of the resolution enhancement [4].

We again turn to simulation to gain insight into the correlation between the enhanced resolution images of a given channel and the nonenhanced images from a higher resolution channel. Plots of the correlation coefficients between the enhanced images and the 37- and $85-\mathrm{GHz}$ nonenhanced images versus iteration number and $\gamma$ are contained in Fig. 4. These curves are similar to those in Fig. 3. The correlation coefficient is maximized at slightly different values of $k$ and $\gamma$, depending on the comparison image. Table IV compares the correlation coefficients between the nonenhanced 37- and $85-\mathrm{GHz}$ channels with the processed images of the other channels. The standard deviation of the difference images is also tabulated. When the $37-\mathrm{GHz}$ channel is used for comparison, the SIR algorithm exhibits higher correlation than the BGI, but somewhat lower than in the nonenhanced case. When the $85-\mathrm{GHz}$ channel is used for comparison, both SIR and BGI offer improved correlation with SIR somewhat higher than BGI.

\section{B. Comments}

As is evident in these simulation results, both the SIR and BGI algorithms can enhance the data and have similar resolution-enhancement capability. However, SIR is computationally less intense than BGI and is, therefore, faster. For example, the SIR-processed simulation images take about one min on an HP workstation, while the BGI $\left(g_{c}=-11\right.$ $\mathrm{dB}$ ) images take approximately $30 \mathrm{~min}$. (Significantly more computation can be required for smaller $g_{c}$ values, which result in larger measurement matrices.) The computational difference comes from basic algorithmic approaches. BGI requires a matrix inversion for each pixel, while SIR requires only relatively simple calculations. We note that when multiple images with exactly the same measurement and pixel locations are processed, the BGI-enhancement coefficients may be stored and reused.

Another difference between BGI and SIR is the use of tuning parameters to tradeoff noise and resolution. The $\gamma$ coefficient in the BGI algorithm explicitly provides a tradeoff between 


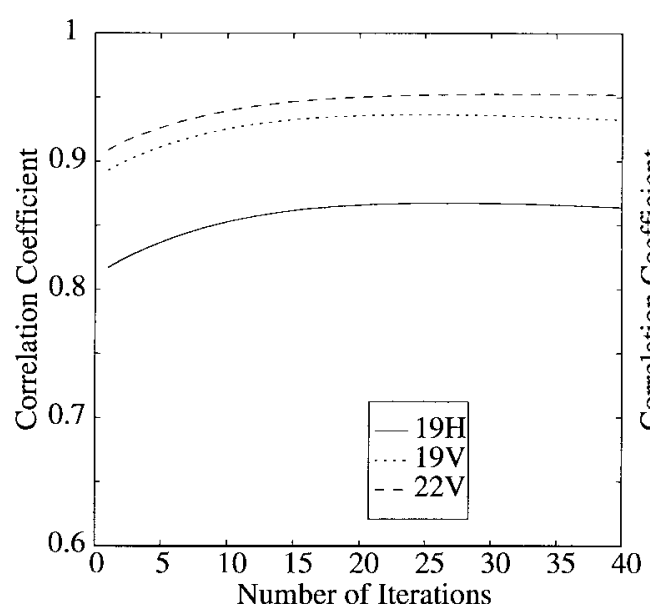

(a)

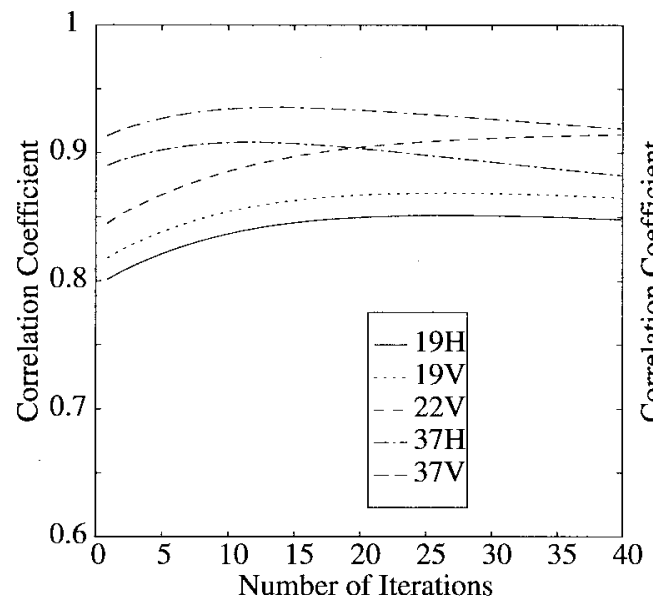

(c)

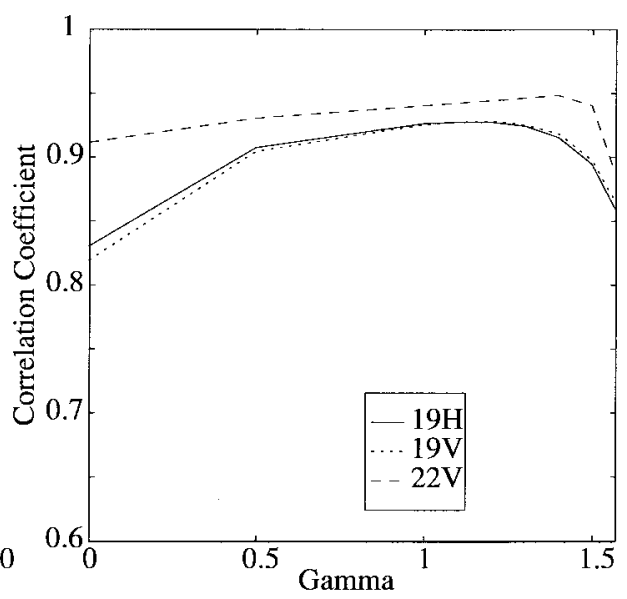

(b)

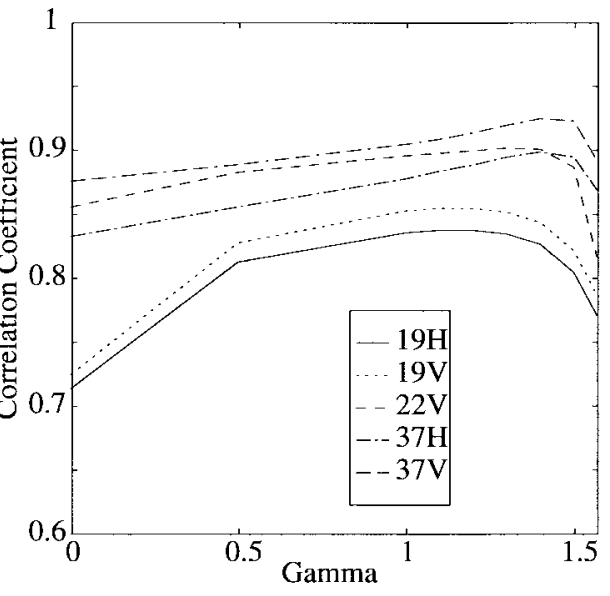

(d)

Fig. 4. Correlation coefficient between simulation results and nonenhanced images. (a) SIR $19 \mathrm{~V}$ and nonenhanced $37 \mathrm{~V}$ versus SIR iteration number $k$, (b) BGI $19 \mathrm{~V}$ and nonenhanced $37 \mathrm{~V}$ versus BGI $\gamma$ coefficient, (c) SIR $19 \mathrm{~V}$ and nonenhanced $85 \mathrm{~V}$ versus SIR iteration number $k$, and (d) BGI $19 \mathrm{~V}$ and nonenhanced $37 \mathrm{~V}$ versus BGI $\gamma$ coefficient.

TABLE IV

Standard Deviation of the Difference and the Correlation Coefficient Between Simulated Processed Images and Nonenhanced Images of the Corresponding Polarization. $N$ and $\gamma$ Values from table III Are Used. No Ocean or River Pixels Are Included in the Statistics Calculation

\begin{tabular}{c|c|c|c|c|c}
\hline \multirow{2}{*}{ Channel } & & \multicolumn{2}{|c|}{$37 \mathrm{GHz}$ Comparison } & \multicolumn{2}{c}{$85 \mathrm{GHz}$ Comparison } \\
\hline $19 \mathrm{H}$ & Algorithm & Std (K) & Correlation Coef. & Std (K) & Corrclation Coef. \\
& non & 1.063 & 0.962 & 2.609 & 0.796 \\
& SIR & 2.080 & 0.868 & 2.258 & 0.851 \\
& BGI & 1.394 & 0.927 & 2.361 & 0.836 \\
\hline $19 \mathrm{~V}$ & non & 1.101 & 0.958 & 2.453 & 0.812 \\
& SIR & 1.301 & 0.937 & 2.080 & 0.868 \\
& BGI & 1.392 & 0.926 & 2.193 & 0.853 \\
\hline $22 \mathrm{~V}$ & non & 0.855 & 0.978 & 2.140 & 0.866 \\
& SIR & 1.175 & 0.953 & 1.703 & 0.914 \\
& BGI & 1.166 & 0.949 & 1.838 & 0.901 \\
\hline $37 \mathrm{H}$ & non & & & 2.160 & 0.865 \\
& SIR & & & 1.809 & 0.907 \\
& BGI & & & 1.900 & 0.899 \\
\hline $37 \mathrm{~V}$ & non & & & 1.897 & 0.892 \\
& SIR & & & 1.515 & 0.933 \\
& BGI & & & 1.609 & 0.925 \\
\hline
\end{tabular}

noise and resolution. Although a tradeoff between noise and resolution can be made via the number of iterations used for SIR, there is no explicit relationship between the noise variance and the number of iterations required.
It should be noted that when multiple passes are combined, further resolution enhancement and noise reduction are possible with SIR, as done with scatterometer data [12]. The multiple passes permit, in effect, averaging of the measure- 
ments on the pixel grid with consequent noise reduction. Of course, to be effective, the surface must be constant from pass to pass. As currently formulated, it is difficult to use multiple passes with BGI, due to the occurrence of singular matrices in the measurement matrix inverted as part of the algorithm. The singularity arises when several measurements occur at essentially the same location. Noise in these multiple measurements gives rise to an inconsistent linear system, which can result in a singular matrix.

In summary, based on simulation, SIR and BGI offer similar resolution enhancement. However, SIR requires much less computation and can be used with multiple-pass data. Using the $\gamma$ coefficient, BGI offers a subjective tradeoff between noise and resolution. A similar tradeoff is possible with SIR by limiting the number of iterations.

\section{ACTUAL SSM/I DATA WITH THE BGI AND SIR ALGORITHMS}

Having examined simulated images, we now consider actual data to compare algorithm performance. Since our primary interest in using these algorithms is for land and vegetation imaging, we consider two illustrative examples over South America. The two selected study regions have different radiometric signatures. The Amazon River Delta region includes both ocean and river, which are both much cooler than vegetated areas. The Guyana Highlands region has little surface water, but exhibits significant brightness variations, due to spatial variations in the vegetation coverage. Each example is generated from a different descending SSM/I pass from the F10 satellite during September 1992. As in the simulation, the nonenhanced comparison images are generated by assigning the value of the nearest measurement to each pixel. All images are 32 pixels/deg.

\section{A. Amazon River Delta}

The study area is a $13^{\circ}$ longitude $\times 15^{\circ}$ latitude area over the Amazon River Delta. This region was selected because it contains large regions of relatively homogeneous vegetation along with rivers and coastlines. In the example illustrated here, the 19-GHz V-pol channel is used. The nonenhanced image is shown in Fig. 5(a). The 19-V SIR and BGI images for the "optimum" $N$ and $\gamma$ values from Table III are given in Fig. 5(c) and (d). For comparison purposes, the nonenhanced 37-GHz V image is shown in Fig. 5(b).

The difference in the brightness response of the surface between the 19 and $37 \mathrm{GHz}$ is evidenced by the dark spot in the lower left of the images, which is most evident in the nonenhanced $37-\mathrm{GHz}$ image. This spot is due to an atmospheric effect, such as a cloud or rain. Visually, the BGI and the SIR images are very similar, though there are some processing artifacts along the coast and rivers. Subjectively, the SIR image exhibits the greatest contrast along the rivers where the brightness-temperature step from water to land is very large $(\sim 160-\sim 285 \mathrm{~K})$. The overshoot in the brightness of the land along the river appears strongest in the SIR image.

Fig. 6 plots the correlation between the enhanced images and the nonenhanced $37-$ and $85-\mathrm{GHz}$ images, as a function
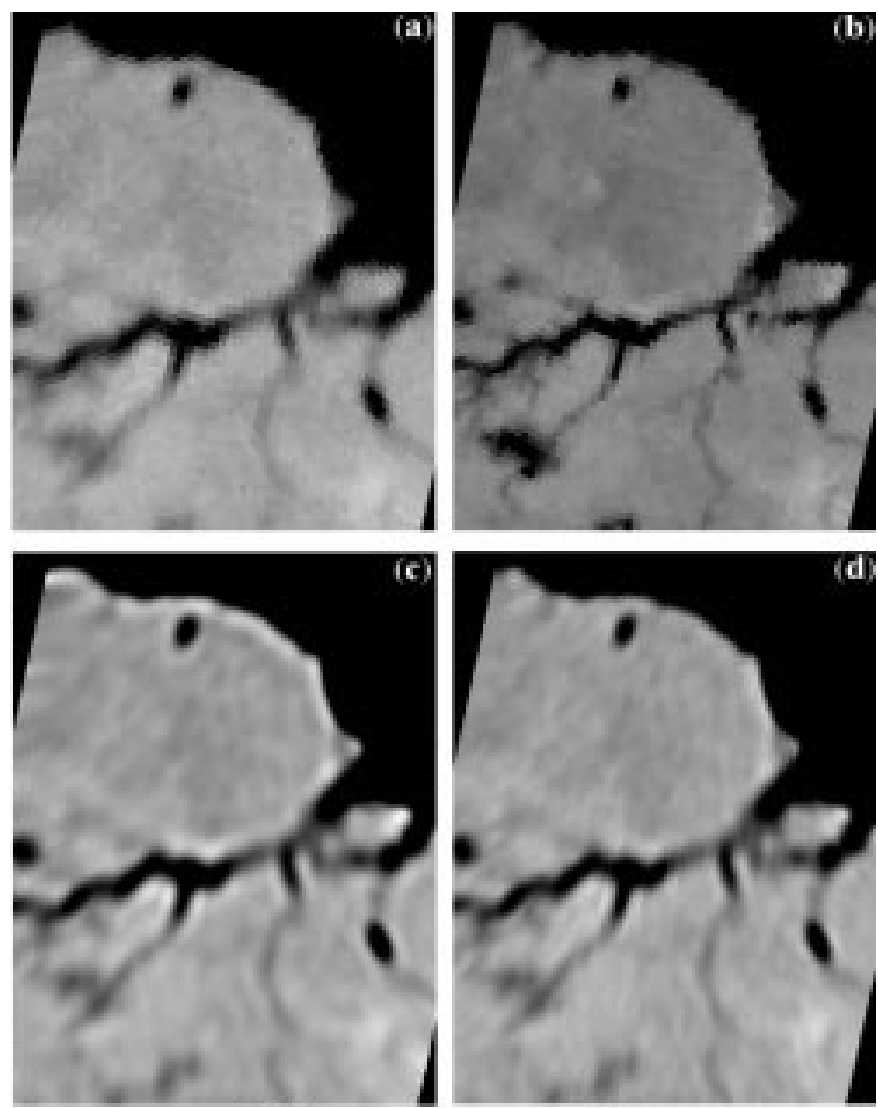

270

\section{0}

Fig. 5. Images of Amazon River Delta region: (a) 19-V nonenhanced, (b) 37-V nonenhanced, (c) 19-V SIR, and (d) 19-V BGI

of $k$ and $\gamma$. The values of $k$ and $\gamma$ that maximize the correlation are similar to those predicted by simulation. We note that near the maximum correlation, the correlation coefficient is not particularly sensitive to the exact value of $k$. Thus, we can use the values chosen in the simulation without significantly altering the correlation. Similar conclusions can be made regarding the $\gamma$ value.

Table $\mathrm{V}$ gives the correlation coefficients between the nonenhanced 37- and $85-\mathrm{GHz}$ images and the processed images from the other channels, using the "optimum" $N$ and $\gamma$ values from the simulation. The standard deviation of the difference between the images is also given. The standard deviation is higher for the $85-\mathrm{GHz}$ channel comparison. This results from large differences in pixel values along the river, due to the fact that the river appears much wider in the lower frequency channels than it appears in the $85-\mathrm{GHz}$ channel. This is because of the higher intrinsic resolution and sampling of the $85-\mathrm{GHz}$ channel.

Using the correlation coefficients in Table V, we see that when the nonenhanced $85 \mathrm{GHz}$ is used for comparison, SIR has a similar or slightly larger correlation than both the BGI and the corresponding nonenhanced images. When the nonenhanced $37-\mathrm{GHz}$ image is used for comparison, the BGI has higher correlation than the $19-\mathrm{GHz}$ SIR, but lower than the 22-GHz SIR. The correlation for both the BGI and the SIR images is lower than the corresponding nonenhanced 


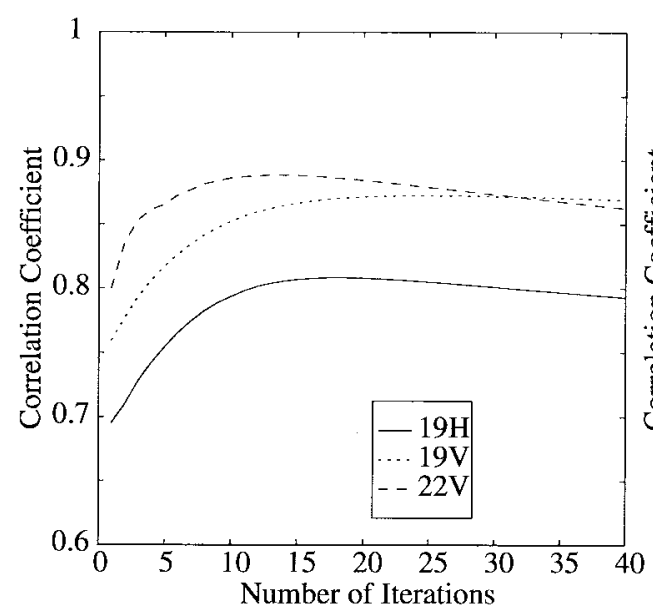

(a)

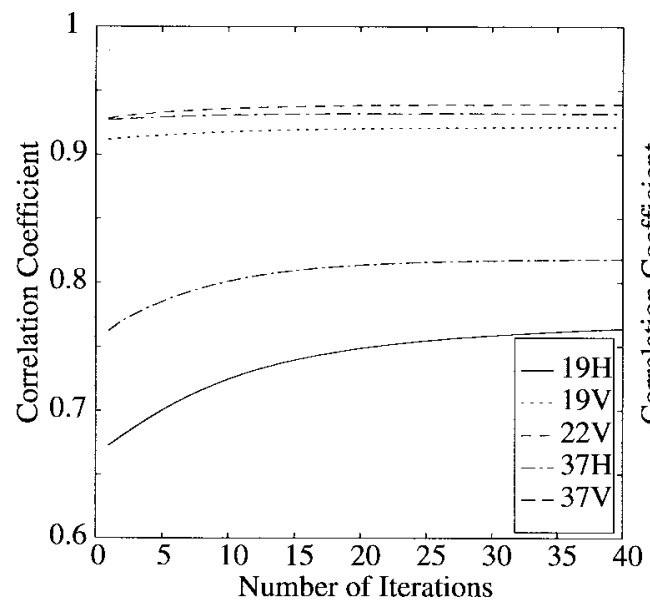

(c)

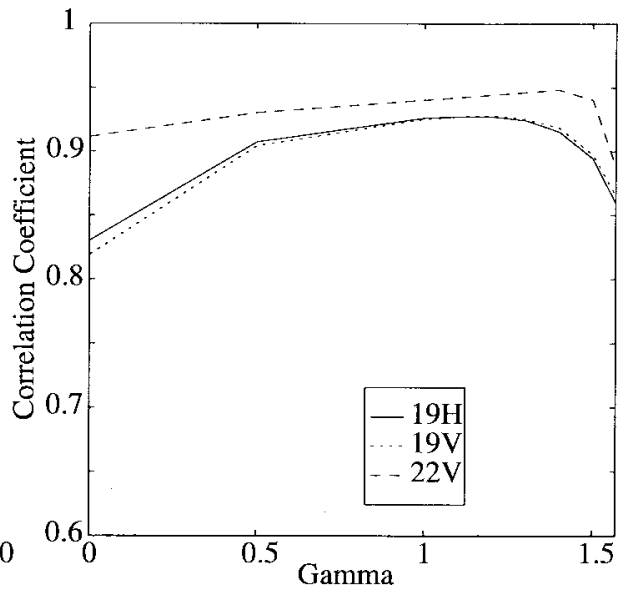

(b)

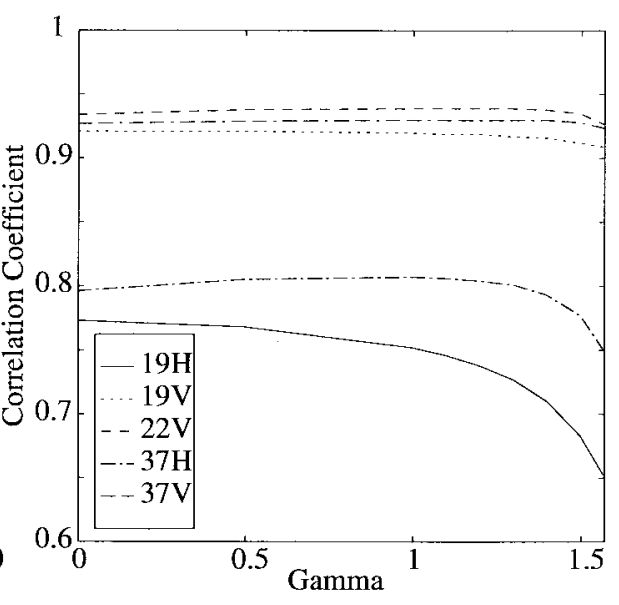

(d)

Fig. 6. Correlation coefficient between Amazon River Delta region results and nonenhanced images: (a) SIR 19 V and nonenhanced 37 V versus SIR iteration number $k$, (b) BGI $19 \mathrm{~V}$ and nonenhanced $37 \mathrm{~V}$ versus BGI $\gamma$ coefficient, (c) SIR $19 \mathrm{~V}$ and nonenhanced 85 V versus SIR iteration number $k$, and (d) BGI $19 \mathrm{~V}$ and nonenhanced $85 \mathrm{~V}$ versus BGI $\gamma$ coefficient.

TABLE V

Standard Deviation of the Difference and the Correlation Coefficient Between Processed Images and Nonenhanced Images of the Corresponding Polarization for the Amazon Delta Region. $N$ and $\gamma$ Values from Table III Are Used. Ocean Pixels Are Excluded in the Statistics Calculation

\begin{tabular}{c|c|c|c|c|c}
\hline \multirow{3}{*}{ Channel } & & \multicolumn{2}{|c|}{37 GHz Comparison } & \multicolumn{2}{|c}{$85 \mathrm{GHz}$ Comparison } \\
\hline $19 \mathrm{H}$ & Algorithm & Std $(\mathrm{K})$ & Correlation Coef. & Std $(\mathrm{K})$ & Correlation Coef. \\
& non & 3.185 & 0.841 & 12.38 & 0.716 \\
& SIR & 3.831 & 0.805 & 13.12 & 0.754 \\
& BGI & 3.603 & 0.818 & 12.57 & 0.752 \\
\hline $19 \mathrm{~V}$ & non & 2.522 & 0.881 & 23.13 & 0.916 \\
& SIR & 2.911 & 0.873 & 23.56 & 0.921 \\
& BGI & 2.789 & 0.873 & 23.43 & 0.920 \\
\hline $22 \mathrm{~V}$ & non & 2.048 & 0.913 & 10.32 & 0.933 \\
& SIR & 2.177 & 0.863 & 10.56 & 0.939 \\
& BGI & 3.417 & 0.667 & 10.45 & 0.938 \\
\hline $37 \mathrm{H}$ & non & & & 10.26 & 0.771 \\
& SIR & & & 10.08 & 0.809 \\
& BGI & & & 9.883 & 0.793 \\
\hline $37 \mathrm{~V}$ & non & & & 17.41 & 0.927 \\
& SIR & & & 17.52 & 0.932 \\
& BGI & & & 17.44 & 0.930 \\
\hline
\end{tabular}

images. Overall, we conclude that BGI and SIR produce similar results and, based on the improved correlation of the enhanced images, both algorithms are effective at resolution enhancement.

\section{B. Guyana Highlands}

A second vegetation region is also considered. The study region is a $6^{\circ}$ longitude $\times 5^{\circ}$ latitude area over the Guyana 
TABLE VI

Standard Deviation of the Difference and the Correlation Coefficient Between Processed Images and Nonenhanced Images of the Corresponding Polarization for the Guyana Highlands Region. $N$ and $\gamma$ Values from Table III Are Used

\begin{tabular}{c|c|c|c|c|c}
\hline & & \multicolumn{2}{|c|}{$37 \mathrm{GHz}$ Comparison } & \multicolumn{2}{c}{$85 \mathrm{GHz}$ Comparison } \\
Channel & Algorithm & Std $(\mathrm{K})$ & Correlation Coef. & Std (K) & Correlation Coef. \\
\hline $19 \mathrm{H}$ & non & 1.635 & 0.728 & 2.339 & 0.438 \\
& SIR & 1.766 & 0.719 & 2.491 & 0.442 \\
& BGI & 1.747 & 0.714 & 2.466 & 0.432 \\
\hline $19 \mathrm{~V}$ & non & 0.948 & 0.964 & 1.451 & 0.887 \\
& SIR & 0.925 & 0.964 & 1.501 & 0.902 \\
& BGI & 0.958 & 0.961 & 1.501 & 0.897 \\
\hline $22 \mathrm{~V}$ & non & 1.315 & 0.938 & 1.204 & 0.899 \\
& SIR & 1.363 & 0.922 & 1.248 & 0.906 \\
& BGI & 1.626 & 0.867 & 2.239 & 0.663 \\
\hline $37 \mathrm{H}$ & non & & & 1.485 & 0.795 \\
& SIR & & & 1.474 & 0.803 \\
& BGI & & & 1.393 & 0.814 \\
\hline $37 \mathrm{~V}$ & non & & & 1.601 & 0.897 \\
& SIR & & & 1.639 & 0.907 \\
& BGI & & & 1.487 & 0.908 \\
\hline
\end{tabular}
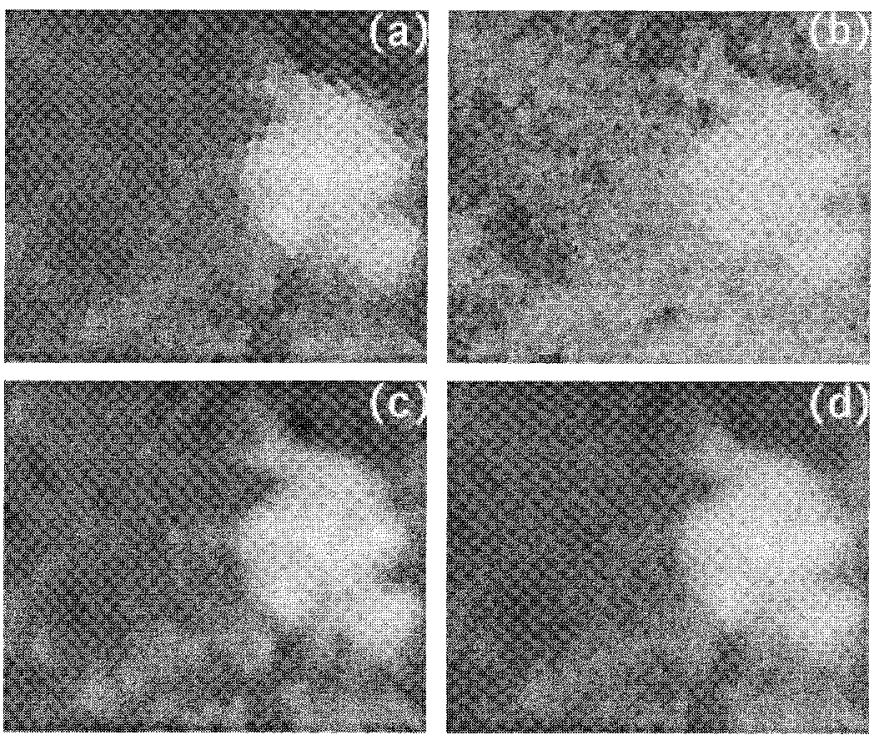

270 300

Fig. 7. Images of Guyana Highlands region: (a) $37-\mathrm{V}$ nonenhanced, (b) $85-\mathrm{V}$ nonenhanced, (c) 37-V SIR, and (d) 37-V BGI.

Highlands of South America. In this scene, the brightnesstemperature step from the lowland tropical forest to the grassy highland region is much smaller $(280-\sim 285 \mathrm{~K})$ than the river to forest step in the Amazon River Delta region images.

For the image examples, the $37-\mathrm{GHz} \mathrm{V}$-pol channel is used. The nonenhanced 37-GHz image is shown in Fig. 7(a). Fig. 7(c) shows the SIR image, while (d) shows the BGI image. For comparison, the nonenhanced $85-\mathrm{GHz} \mathrm{V}$-pol images is shown in (b). Though the SIR and the BGI images are very similar, edges are more clearly delineated in the SIR image and then in the BGI image, and there is somewhat more detail in the various regions than in the nonenhanced image. Some processing artifacts are visible in the BGI image in the bright highland region. Table VI gives the correlation coefficients between the nonenhanced $37-$ and $85-\mathrm{GHz}$ images and the processed images from the other channels. The standard devi- ation of the difference between the images is also given. As in the Amazon region case, the SIR provides somewhat better correlation than the BGI, when the $85-\mathrm{GHz}$ channel is used for comparison. Though not shown here, plots of the correlation versus $k$ and $\gamma$ for this region are similar to the results from the Amazon River Delta region shown in Fig. 6.

Considering both the image and the correlation coefficient results for the actual SSM/I data in these study regions, we conclude that BGI and SIR produce similar results and are effective for resolution enhancement. Because of its reduced computational requirements, however, we prefer the SIR algorithm.

\section{CONCLUSION}

A comparison of two different methods, BGI and SIR, for improving the resolution of SSM/I images over land regions has been presented. The algorithms have similar resolutionenhancement capability, based on simulation and results from actual SSM/I data. BGI permits explicit tradeoffs between resolution enhancement and noise reduction. A tradeoff between noise and resolution is possible for SIR by varying the number of iterations. The most significant difference between the algorithms is computation: SIR requires less than $1 / 30$ of the computation required for BGI. In addition, SIR can also be used with multiple passes to reduce the image noise level. The enhanced resolution SSM/I images better resolve small features and have a higher definition of coastlines and other edges than the nonenhanced images. For example, improved resolution SSM/I data has been shown to be effective in iceconcentration estimates near the ice edge [17]. Images will also be useful in land and vegetation studies.

\section{ACKNOWLEDGMENT}

The SSM/I data used in the paper was made available courtesy of Dr. F. Wentz at Remote Sensing Systems [21]. The authors wish to thank Q. Remund for his help in processing the data. 


\section{REFERENCES}

[1] G. E. Backus and J. F. Gilbert, "Numerical applications of a formalism for geophysical inverse problems," Geophys. J. R. Astron. Soc., vol. 13, pp. 247-276, 1967.

[2] - "Resolving power of gross Earth data," Geophys. J. R. Astron. Soc., vol. 16, pp. 169-205, 1968.

[3] B. Caccin, C. Roberti, P. Russo, and A. Smaldone, "The Backus-Gilbert inversion method and the processing of sampled data," IEEE Trans. Signal Processing, vol. 40, pp. 2823-2825, Nov. 1992.

[4] M. R. Farrar and E. A. Smith, "Spatial resolution enhancement of terrestrial features using deconvolved SSM/I brightness temperatures," IEEE Trans. Geosci. Remote Sensing, vol. 30, pp. 349-355, Mar. 1992.

[5] N. C. Grody, "Classification of snow cover and precipitation using the special sensor microwave/imager," J. Geophys. Res., vol. 94, pp. 7423-7435, Apr. 1991.

[6] J. P. Hollinger, J. L. Pierce, and G. A. Poe, "SSM/I instrument evaluation,” IEEE Trans. Geosci. Remote Sensing, vol. 28, pp. 781-790, Sept. 1990.

[7] J. P. Hollinger et al., Special Sensor Microwave/Imager User's Guide, Naval Res. Lab., Washington, DC, Dec. 1987.

[8] J. P. Hollinger, DMSP Special Sensor Microwave/Imager Calibration/Validation, Final Report, vol. 1, Naval Res. Lab., Washington, DC, July 1989.

[9] T. J. Jackson and T. J. Schmugge, "Algorithm for the passive microwave remote sensing of soil moisture," in Microwave Radiometry and Remote Sensing Applications, P. Pampaloni, Ed. Zeist, The Netherlands: VSP, 1989, pp. 3-17.

[10] A. K. Jain, Fundamentals of Digital Image Processing. Englewood Cliffs, NJ: Prentice-Hall, 1989.

[11] A. S. Jones and T. H. V. Haar, "Passive microwave sensing of cloud liquid water over land regions," J. Geophys. Res., vol. 95, pp. 16673-16683, Sept. 1990.

[12] D. G. Long, P. J. Hardin, and P. T. Whiting, "Resolution enhancement of spaceborne scatterometer data," IEEE Trans. Geosci. Remote Sensing, vol. 31, pp. 700-715, May 1993.

[13] M. J. McFarland, R. L. Miller, and C. M. U. Neale, "Land surface temperature derived from the SSM/I passive microwave brightness temperatures," IEEE Trans. Geosci. Remote Sensing, vol. 28, pp. 839-845, Sept. 1990.

[14] P. Pampaloni and S. Paloscia, "Microwave emission and plant water content: A comparison between field measurement and theory," IEEE Trans. Geosci. Remote Sensing, vol. GE-24, pp. 900-904, Nov. 1986.

[15] G. A. Poe, "Optimum interpolation of imaging microwave radiometer data," IEEE Trans. Geosci. Remote Sensing, vol. 28, pp. 800-810, Sept. 1990.

[16] W. D. Robinson, C. Kummerow, and W. S. Olson, "A technique for enhancing and matching the resolution of microwave measurements from the SSM/I instrument," IEEE Trans. Geosci. Remote Sensing, vol. 30, pp. 419-429, May 1992

[17] R. Sethmann, B. A. Burns, and G. C. Heygster, "Spatial resolution improvement of SSM/I data with image restoration techniques," IEEE Trans. Geosci. Remote Sensing, vol. 32, pp. 1144-1151, Nov. 1994.

[18] A. Stogryn, "Estimates of brightness temperatures from scanning radiometer data," IEEE Trans. Antennas Propagat., vol. AP-26, pp. $720-726$, Sept. 1978

[19] R. H. Thomas, R. A. Bindschadler, R. L. Cameron, F. D. Carsey, B Holt, T. J. Hughes, C. W. M. Swithinbank, I. M. Whillans, and H. J. Zwally, "Satellite remote sensing for ice sheet research," NASA Tech. Memo. 86233, 27, 1985.

[20] F. T. Ulaby, R. K. Moore, and A. K. Fung, Microwave Remote SensingActive and Passive. vol. 1. Norwood, MA: Artech House, 1981.

[21] F. J. Wentz, User's Manual SSM/I Antenna Temperature Tapes. Remote Sensing Syst., Santa Rosa, CA, 1991.

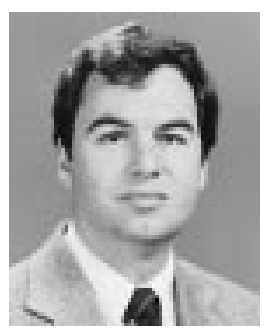

David G. Long (S'80-M'82) received the Ph.D. degree in electrical engineering from the University of Southern California, Los Angeles, in 1989.

From 1983 to 1990 , he was with NASA's Jet Propulsion Laboratory (JPL), Pasadena, CA, where he developed advanced radar remote-sensing systems. While at JPL, he was the Senior Project Engineer on the NASA Scatterometer (NSCAT) project. He is currently an Associate Professor in the Electrical and Computer Engineering Department at Brigham Young University, Provo, UT, where he teaches upper division and graduate courses in communications, microwave remote sensing, radar, and signal processing. He is the Principal Investigator on several NASA-sponsored interdisciplinary research projects in remote sensing, including innovative radar systems, spaceborne scatterometry of the ocean and land, and modeling of atmospheric dynamics. He has numerous publications in signal processing and radar scatterometry. His research interests include microwave remote sensing, radar theory, spacebased sensing, estimation theory, computer graphics, signal processing, and mesoscale atmospheric dynamics.

Dr. Long is a member of the NSCAT Science Working Team.

Douglas L. Daum, photograph and biography not available at the time of publication. 